\title{
Task structure variables affecting concept identification
}

\author{
VLADIMIR PISHKIN \\ VA Hospital and University of Oklahoma Health Sciences Center, Oklahoma City, Oklahoma 73104 \\ LYLE E. BOURNE, JR. \\ University of Colorado, Boulder, Colorado 80302 \\ and \\ STEVEN M. FISHKIN \\ VA Hospital and University of Oklahoma Health Sciences Center, Oklahoma City, Oklahoma 73104
}

\begin{abstract}
A mismatch between the number of values of the relevant and the irrelevant dimensions (when there is more than one irrelevant dimension) in a stimulus population enhances concept identification, probably because of a stimulus isolation effect. A mismatch between the number of values of the relevant dimension and the number of response categories, producing a form of S-R incompatibility, inhibits performance. Attentional and hypothesis testing processes appear to underlie both of these effects.
\end{abstract}

In most concept learning experiments, $S$ must discover one or more relevant stimulus dimensions and assign all dimension values or combinations of values uniquely to a corresponding set of response categories (e.g., Pishkin, 1960). Manipulation of problem complexity in these studies is typically accomplished by introducing one or more irrelevant dimensions each with the same number of values as the relevant dimension and as there are response categories. It occurred to the authors that performance in a concept identification task might be affected by mismatches between number of values per relevant dimension, number of values per irrelevant dimension, and number of response categories. For example, $\mathrm{S}$ might find it easier to identify the relevant dimension when the number of its values matches the number of response categories or mismatches the number of values on each irrelevant dimension. A mismatch between the number of values of relevant and irrelevant dimensions might isolate, perceptually or cognitively, the relevant dimension, and a match between relevant dimension and response categories should produce facilitative S-R compatibility. (Fitts \& Seeger, 1953; Kresse, Peterson, \& Grant, 1954).

The general idea of the present experiment was to examine a number of intuitively interesting match-mismatch conditions involving relevant dimensions, irrelevant dimensions, and response categories over a range of problem difficulty. The results obtained were stable, interesting, but puzzling. They lead us to postulate tentatively an interpretation based on implicit attentional and hypothesis testing processes.

\section{METHOD}

\section{Subjects}

The experiment was conducted simultaneously at the University of Colorado, Boulder, (UC) and Oklahoma City
University (OCU). A total of 252 Ss were all female volunteers from introductory psychology classes of the two universities. They participated individually and were assigned at random, but in equal number, to experimental conditions. None of the Ss had previously participated in a concept learning experiment. All Ss were presented with a problem in which form of figure was the relevant dimension.

The Ss were divided into three equal groups on the basis of whether their problem contained one, two, or three irrelevant dimensions. The main variables were the number of values per relevant and irrelevant dimensions and the number of response categ ories. Variations in values and categories are summarized by labels in which the first number represents the number of values on the relevant dimension, the second number represents the number of values per irrelevant dimension, and the third number indicates the number of response categories available to $\mathrm{S}$. Accordingly, the six main conditions can be summarized as follows: $222,232,322,332,323$, and 333 .

\section{Task and Apparatus}

The stimulus patterns were geometric designs, projected on a viewing screen in front of $S$. At most, these patterns varied in four dimensions: color, size, number, and form, each with a maximum of three values per dimension. The single relevant dimension in all problems was form (squares, triangles, and hexagons). Possible values of the irrelevant dimensions were (number) one, two, or three figures, (color) red, yellow, or blue, and (size) large, medium, or small.

The presentation of stimulus patterns, the control of feedback signals (1 sec duration) and of overall interstimulus interval $(6 \mathrm{sec})$, and the recording of responses and feedback were accomplished automatically by an apparatus described earlier (Pishkin, 1960).

At the beginning of the experiment, each $S$ received oral instructions describing the nature of a concept, the general procedure of the experiment, the use of the response buttons, and the meaning of the feedback. The S's problem was to learn the required association between two (or three) response categories (pushbuttons) and the two (or three) attributes of a single relevant dimension. After $S$ pushed a button to indicate in which category he felt the stimulus pattern belonged, the stimulus screen became blank and a light went on over the correct response button. The next stimulus appeared on the screen $6 \mathrm{sec}$ later, and the cycle was repeated. All conditions 
Table 1

Mean Errors as a Function of Values on Relevant (R), Irrelevant (I) Dimensions, and Response Categories

\begin{tabular}{|c|c|c|c|}
\hline \multirow[b]{2}{*}{ Condition } & \multicolumn{2}{|c|}{ Dimensions } & \multirow[b]{2}{*}{ Overall } \\
\hline & 1 & 2 and 3 & \\
\hline $2 \mathrm{R}$ by $2 \mathrm{I}$ by $2 \mathrm{C}$ & 1.02 & 4.94 & 3.63 \\
\hline $2 \mathrm{R}$ by $3 \mathrm{I}$ by $2 \mathrm{C}$ & 4.22 & 3.26 & 3.58 \\
\hline $3 R$ by $2 \mathrm{I}$ by $2 \mathrm{C}$ & 2.11 & 5.17 & 4.15 \\
\hline $3 R$ by $3 I$ by $2 C$ & 2.76 & 7.05 & 5.62 \\
\hline $3 R$ by $2 \mathrm{I}$ by $3 \mathrm{C}$ & 1.72 & 2.98 & 2.52 \\
\hline $3 R$ by $3 I$ by $3 C$ & 3.18 & 6.58 & 5.45 \\
\hline
\end{tabular}

were self-paced in that $\mathrm{S}$ was allowed as much time as he needed to respond to any pattern. When $S$ reached a criterion of 16 correct responses in a row, her session was terminated.

\section{RESULTS AND DISCUSSION}

The results obtained from UC and OCU were virtually identical, despite differences in Es, Ss, and other minor procedural factors (all Fs $\sim 1.0$ ). Therefore, the most efficient way to present the results appears to be in the form of a single analysis of pooled data. Errors to solution were cast into a 6 by 3 analysis of variance, with six value-category conditions and three levels of problem complexity (number of irrelevant dimensions). Over the entire set of experiments, 14 Ss contributed to each of the 18 treatment groups. In this analysis, the main effects of both variables were statistically reliable: value-categ ory condition, $\mathrm{F}(5,234)=34.87, \mathrm{p}<.01$, and complexity, $\mathrm{F}(2,234)=5.23, \mathrm{p}<.01$. The major difference among complexity conditions lay in the comparison of one irrelevant dimension and more than one. Therefore, in Table 1, mean error scores for each of the six main groups are presented separately for one and the combination of two and three irrelevant dimensions.

As suggested earlier, relationships among three variables, the number of values per relevant dimension, the number of values per irrelevant dimension, and the number of available response categories, appear likely to contribute to potential stimulus dimension isolation and to S-R compatibility effects in concept identification. These variables relate to three match-mismatch hypotheses. (A) Isolation of the relevant dimension exists when there is a mismatch between the number of values on the relevant and the irrelevant dimensions. Isolation of the relevant dimension should facilitate performance, although this effect should hold only when there is more than one irrelevant dimension. (B) A form of S-R compatibility is obtained when the number of levels per relevant dimension matches the number of available response categories. Compatibility should facilitate processing of relevant information, and this effect should hold regardless of the number of irrelevant dimensions. (C) When the number of levels of the irrelevant dimensions match the number of response categories, a compatibility exists between the two which is counterproductive. Performance should be poorer when there is a match between number of levels per irrelevant dimension and number of response categories, and this effect should occur regardless of the number of irrelevant dimensions.

Data bearing on these hypotheses are presented in Table 2. As can be seen, Hypothesis A is supported, Hypothesis B is supported when there are two or three irrelevant dimensions, and Hypothesis $\mathrm{C}$ is supported numerically but not statistically. These data suggest an ordering of match-mismatch conditions in terms of the magnitude of their performance effects. Isolation of the relevant dimension generated by a mismatch of values with the irrelevant dimensions is the most potent factor. Somewhat less important is a match between the number of values per relevant dimension and number of available response categories. The mismatch between the number of available response categories and number of values per irrelevant dimension is marginally effective, at best.

\section{The Isolation Effect}

The isolation effect, generated in this experiment by a mismatch in the number of values on the relevant and irrelevant dimensions, may derive from a two-step process. First, $\mathrm{S}$ organizes the stimulus material into conceptual units; second, he selects one of the units as a source of dimensional hypotheses to test. One basis for forming conceptual units is identity in number of levels of the various stimulus dimensions. Those dimensions

Table 2

An Evaluation of Hypotheses Concerning Matches and Mismatches Between (I) Number of Levels of Relevant Dimensions,

(II) Number of Levels on Irrelevant Dimensions, and (III) Number of Response Categories

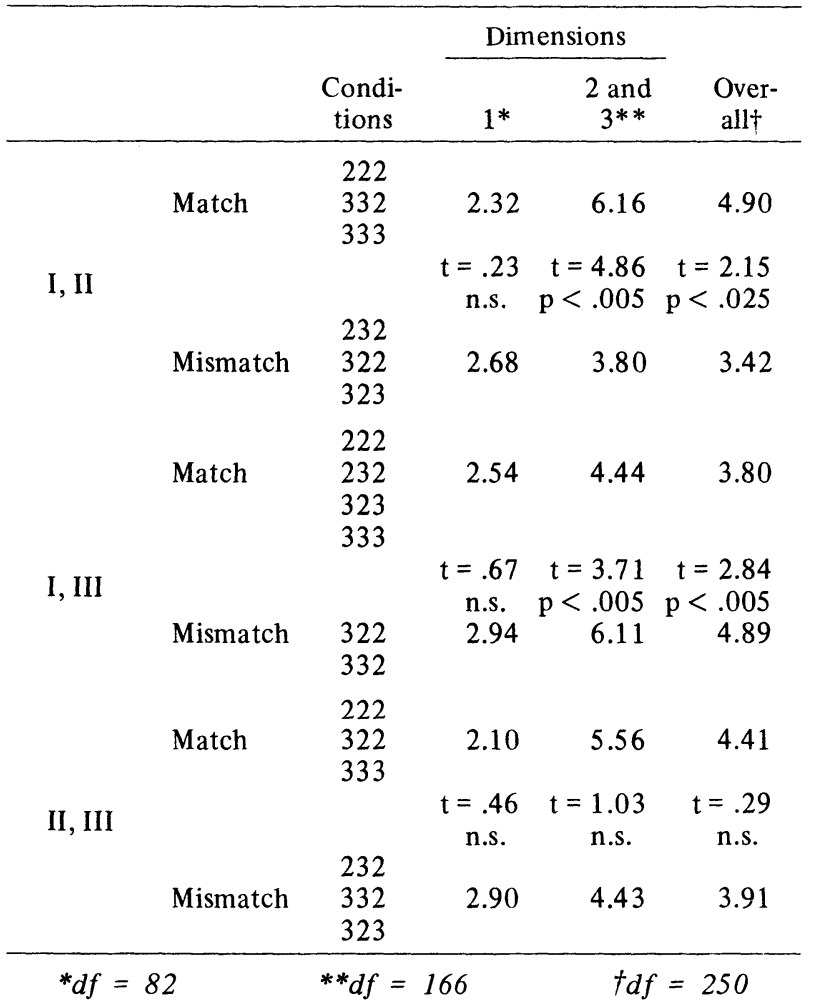


which have like numbers of levels are grouped, the two-leveled dimensions in one class and the three-leveled dimensions in another. Selection of a focal dimension within one of these units appears to be made largely on the basis of simplicity or uniqueness. That is, when the organizational process yields one category with a smaller number of stimulus dimensions, specifically when a category contains only one stimulus dimension, dimensional selection will be made on that category until its contents have been tested and rejected.

\section{Compatibility}

Compatibility between levels of the relevant dimension and response categories enhances learning. A corollary, that S-R compatibility involving the irrelevant dimension should hinder learning, is not supported by the data. It seems intuitively clear that $S$ should be more likely to test first as possible solutions those dimensions which are compatible with the response options. In effect, this reasoning postulates that compatibility operates on any dimension, relevant or irrelevant, such that attention is directed toward compatible dimensions and away from incompatible ones. Thus, when the irrelevant dimensions lacked S-R compatibility, they would in general not be tested first, thereby favoring early testing of the relevant dimension. Since the obtained results do not support the hypothesis, it may be presumed that additional factors are involved.

Our present thinking suggests that both attentional and hypothesis testing processes underlie S-R compatibility effects. With respect to relevant dimensions, the two processes are additively facilitative of performance. In the case of irrelevant dimensions, the two processes are activated antagonistically and tend to of fset each other. Table 3 outlines these ideas. The plusses in the upper left quadrant are interpreted as follows: attentional and hypothesis testing mechanisms are both facilitated when S-R compatibility obtains for the relevant dimension. The $S$ attends to the relevant dimension, and its hypotheses are simply evaluated.
Table 3

Effects of S-R Compatibility on Hypothesized Attentional and Hypothesis Testing Processes in CI

\begin{tabular}{|c|c|c|c|c|}
\hline & \multicolumn{2}{|c|}{$\begin{array}{c}\text { Response } \\
\text { Compatibility }\end{array}$} & \multicolumn{2}{|c|}{$\begin{array}{c}\text { Response } \\
\text { Incompatibility }\end{array}$} \\
\hline & $\mathrm{Att}^{*}$ & $\mathrm{HT} \dagger$ & Att & HT \\
\hline Relevant Dimension & + & + & - & - \\
\hline Irrelevant Dimension & - & + & + & - \\
\hline
\end{tabular}

${ }^{*}$ Att $=$ attentional effect

${ }^{+} H T=$ hypothesis testing

Under conditions of incompatibility, $\mathrm{S}$ is as likely (incompatible irrelevant dimensions) or more likely (compatible irrelevant dimensions) to attend to irrelevant dimensions first. When he does attend to the relevant dimension, hypothesis testing is relatively difficult. When S-R compatibility obtains for irrelevant dimensions, $S$ attends to them, thus interfering with rapid problem solving, but hypothesis tests are straightforward, leading to easy rejection of irrelevant hypotheses. Under conditions of incompatibility, $\mathrm{S}$ is less likely to attend to irrelevant dimensions, but, if he does, hypothesis testing will be relatively difficult.

Thus, whether or not the relevant dimension of concept exhibits S-R compatibility will have significant effect on performance, whereas this difference will be relatively inconsequential for irrelevant dimensions. While this analysis is post hoc, it is entirely consistent with the results obtained. We plan other experiments to provide a direct a priori test of these ideas.

\section{REFERENCES}

Fitts, P. M., \& Seeger, C. M. S-R compatibility: Spatial characteristics of stimulus and response codes. Journal of Experim ental Psychology, 1953, 46, 199-210.

Kresse, F. H., Peterson, R. M., \& Grant, D. A. Multiple response transfer as a function of supplementary training with verbal schematic aids. Journal of Experimental Psychology, 1954, 48, 381-390.

Pishkin, V. Effects of probability of misinformation and number of irrelevant dimensions upon concept identification. Journal of Experimental Psychology, 1960, 59, 371-378.

(R eceived for publication May 17, 1974.) 